\title{
ANALYSIS OF DRIVER BEHAVIOR IN VEHICLE FOLLOWING AND LANE CHANGING PARAMETERS ON URBAN MID BLOCK SECTIONS
}

\author{
Sowjanya ${ }^{1}$, Dr. S. Moses Santhakumar ${ }^{2}$ \\ ${ }^{1}$ Research Scholar, National Institute of Technology, Civil Engg. Dept., Tiruchirapalli, India \\ ${ }^{2}$ Professor, Civil Engg. Dept., National Institute of Technology, Tiruchirapalli, India
}

\begin{abstract}
In mixed traffic situations, there is weak or no lane behavior of the driver much more complicated where vehicle and driver behavior show a huge difference between them. Road traffic driving behavior on urban midblock sections is one of the most complex phenomena to be examined particularly in heterogeneous traffic conditions. This is often attributed to the capacity of the road section and the traffic flow features at the macroscopic and microscopic level of a road section. Very few researchers have attempted to investigate these features in heterogeneous environments because of the lack of adequate information gathering methods and the amount of complexity involved. In this background, an access controlled mid block road section was selected for video data collection. The main objectives of this study include developing vehicular trajectory data and analyzing the lane changing and vehicle following behavior of driver on the mid block section considering the relative velocities and relative spacing between various types of vehicles under heterogeneous traffic conditions. The videos were collected from urban roadway in the Kurnool district of Andhra Pradesh. The length of the stretch is $120 \mathrm{~m}$ and the width is $7.0 \mathrm{~m}$. The data was extracted to know the variations in terms of longitudinal and lateral speeds, velocities, vehicle following and lane changing behavior of the drivers. The data extracted was smoothened by moving average method to minimize the human errors. Lateral amplitude of the vehicles of various types was analyzed. The study revealed that vehicles in the mixed stream, in general and in particular, Bikes and Autos particularly move substantially in the lateral direction.
\end{abstract}

\section{Keywords: Driver, Vehicle, Urban Mid Block}

\section{Introduction}

The traffic in India is incredibly complex, with little or no lane control. The car not only communicates with the vehicle in front of it, but it also interacts with the vehicles in its immediate proximity. Traffic flow is greatly affected by lane changes. Driving on road sections is one of the most complicated phenomena to be studied, particularly on urban roads, on mid-block sections under mixed traffic conditions.
An Urban Road is a road located within the boundaries of a built-up area. Lane changing behavior is much more complex in mixed traffic conditions, where the behaviors of vehicle and driver show a massive change compared to the behavior under homogeneous traffic conditions. During a lane changing maneuver, small sized vehicles can be easily maneuvered and tend to change lanes using smaller gaps. The lane change duration and the space gaps required for lane changing may also depend on vehicle-type dependent characteristics. The absence of lane behavior implies that a driver not only interacts longitudinally with the vehicles ahead but also laterally with vehicles on either side.

Macroscopic impacts on traffic flow result from aggregate results of microscopic driver behavior. Microscopic driver behavior includes acceleration, deceleration, carfollowing, lane changing behavior, and gap acceptance. Non lane based movement is an indication of weak lane discipline of vehicles, which is predominant in mixed traffic conditions and it is a very important aspect in lane maintenance behavior of mixed traffic. The development of suitable models, to represent the typical behavior of drivers on mid-block sections will be of much practical importance. One of the main conditions for lane discipline is the car or vehicle following theory and the lane changing behaviour of driver. Road safety in the developed world is a big concern. The growing population has raised the traffic risk especially in developing countries like India, as the system is unable to manage and control the increasing vehicular traffic.

\section{Objectives}

- Developing vehicular trajectory data at the mid-block study locations under mixed traffic conditions

- Analysing the Longitudinal and lateral behaviour of driver on the mid block sections in mixed traffic conditions

- To determine the time to collision in vehicle following and lane changing

The paper is divided into six sections, one of which is this one. Section two contains a review of the literature as well as an explanation of the gaps. Section 3 explains the data collection process, approach, and study area description. The fourth section discusses the driver's lane-changing behavior. The section is divided into subsections that characterize lane 
changing duration, vehicle lateral position, and lateral amplitude. Section five discusses the driver's car following behavior in terms of relative velocities, distances, and time to collision. The paper's summary and conclusions are reported in section six.

\section{Literature Review}

In order to investigate vehicle behavior under mixed traffic conditions it is important to know the trajectory details of the vehicle. Different techniques were used in previous works to know the trajectory of the vehicles. In mixed traffic area, Narayana raju et al. (2018) studied vehicle behavior. They studied the macroscopic and microscopic flow properties, including relative speed, and comparatively longitudinal and lateral distance between the vehicles. They concluded that the vehicle's lateral behavior, and its patterns, are not influential in the homogeneous traffic on several highways, play a vital role in driving. Pallav Kumar et al. (2017) extracted the data trajectory from the urban portion of the mid-block and smoothed down the track data extracted to reduce human errors by moving average method, the local weight regression method and the Savitzky Golay filtering method and conducted SWOT analysis. They found that 'moving average method' is the best approach for smoothening vehicle trajectories.

Few studies have looked at the need for lateral movement in various vehicles. The lane change acceptance and duration carried out by Minming Yang et al. (2019). In this study an automatic extraction algorithm was used to test the lane change time of the naturalist driving study. The influence on the following driver also is evaluated and found that the lane change between different types of vehicles ranges from 0.7 seconds to 16.1 seconds. Danish Farooq (2019) examined the lane change effect on a two-lane freeway section from the major traffic parameters. In developing countries, traffic conditions are distinguished by two distinct phenomena. A combination of truck types is the first, and a lack of lane discipline is the second. Anuj Kishor Budhkar and Akhilesh Kumar Maurya (2017) tested different instrumented vehicles of various types with ultrasonic sensors mounted on both sides of the vehicle, which provide inter-vehicular lateral distance and relative speed; and a GPS system fitted with cameras, which provides vehicle position and speed of interacting vehicles. They are driven with varying speeds on different highways in six Indian cities to test the lateral distances kept by multiple interacting vehicles.

Gowri Asaitambi et al. (2018) have studied and developed a model on vehicle lateral shift time, considering the available space gaps, speeds, nearby vehicles and vehicle lateral shifts under medium flow conditions. According to the results, the time it takes to change lanes ranges from 0.15 to 15 seconds, with an average of 2 seconds. the study shows that the right side movement $(2.5 \mathrm{sec})$ of vehicles is somewhat larger than the left side move(2.3 sec). Munigety et al. (2014) conducted an analysis on lane changes on six lane highway in Mumbai using MATLAB programming, which revealed that $2 \mathrm{~W}$ ( $3 \mathrm{sec}$ ) lateral time of movement is four times lesser than that of $3 \mathrm{~W}(12 \mathrm{sec})$.

In the process of evaluating the vehicle following behavior, Venkatesan Kanagaraj and Gowri Asaithambi (2016) observed that vehicles in the mixed traffic conditions, bikes in particular are travelling laterally unsubstantially. In order to reduce human errors during data extraction to a minimum, Chakradhar Reddy et al., (2017), supported the extracted vehicular trajectory data on homogeneous traffic conditions and proposed that Moving Average is the most appropriate approach. The lateral gap was observed by Bangarraju and Ravi Shankar (2016) in mixed traffic conditions with little lane discipline. They concluded that the changing frequency of the Lane is affected by the traffic density, flow and mean speed. Lane is not very independent from lane vehicle arrivals. Mohd Erwan Sanik et al. (2016) used gap acceptation methodology to work on drivers lane changing behaviour and concluded that the main component in lane changing situations is the flow rate of vehicles. In the sense of the gap acceptance approach, Mohd Erwan Sanik et al(2016) studied vehicle lane changes at urban intersections. They also concluded that the key factor affecting the change in lane is the flow rate. Geethi Mukta Mahapatra et al.,(2013) studied the vehicles lateral movements on non lane discipline traffic stream, on a straight road and concluded that lateral acceleration and heading angles are high, in the case of three-wheelers than in case of Cars. The variation of lateral acceleration and heading angles are high at lower speeds. Daniel (Jian) Sun, studied the driver behavior characteristics considering focus group studies and in-vehicle driving tests and developed a gap acceptance algorithm to model lane changing on urban arterials. CORSIM was used to develop the model. C. Mallikarjuna et al., have done research on lateral gaps maintained by different types of vehicles under different traffic conditions. The statistical analysis was done and the gaps maintained by the vehicles with more are less same speeds were normalized.

Time to collision for longitudinal behavior of driver was also carried out by some researchers and is varies from $0.5 \mathrm{sec}$ to $90 \mathrm{sec}$ depending on relative distance and velocity. In the case of opening the mid-range section, rear and side swipe collisions are more common. Simulated driving simulation tests have been conducted by Nengchao et al (2020). The analysis of variance test to assess driving characteristics including TTC was carried out in this study. They found that when shifting the lane from the opening section to the inner lane, drivers can usually control the distance and speed difference from the previous vehicle. Qiang Luo et al (2020) analysed various driver types and quantified the impact on road safety by setting the new car 
rear end collision model. They used fuzzy theory to develop the reasoning model, which took into account conventional safety factors like driver age and fatigue level and output the driver reaction time. They came to the conclusion that keeping a safe distance of 50 meters between the leading and following vehicles would prevent rear-end collisions. Chang Wang et al (2020) developed a lane shifting decision model with a 2-level safe threshold in mixed traffic situations that took into account rear vehicle deceleration behavior. The safe thresholds were determined to be $0.85 \mathrm{~m} / \mathrm{sec}^{2}$ and $1.76 \mathrm{~m} / \mathrm{sec}^{2}$, respectively, based on risk perception of different drivers. Quingwan xue et al. (2018) created a model of rear end collision avoidance behaviour that takes into account driver differences during the car following and collision avoidance process. A Mote Carlo approach was used in model simulation and found that the likelihood of rear-end collisions increased considerably with driving if the distance maintained by subsequent cars dropped under $15 \mathrm{~m}$. TTC is one of the most relevant safety indicators based on time in road safety assessments for the detection of rear end conflicts. Mohmoud Safferzadeh et al (2015) suggested a generalised TTC formulation using motion equations which could be used according to availability of data and the necessary level of accuracy. They came to the conclusion that the TTC ranges between 0.5 and 10 seconds.

\section{Gaps in the literature}

The experiments were conducted to analyse the driver's safety and lane-changing behavior and to extract the vehicle trajectories on control points, on national highways and on multi-lane roads. The focus group and in-vehicle data may not give accurate results as the group of selected people already knows about the test. On longitudinal and lateral behavior of drivers with safe movement, only a few studies have been found in urban areas. Most of the study works have been carried out in the longitudinal and lateral direction without taking account of safety aspects, i.e. safe distance between vehicles.

\section{Study Area and Methodology}

Kurnool is one of the most populous districts in Andhra Pradesh with heavy traffic flow. Government General Hospital is located at the central part of Kurnool city having heavy traffic provided with road dividers. Traffic flow videos are taken by providing CCTV camera on the foot over bridge located at a height of $8.0 \mathrm{~m}$ from the surface of the road. Traffic is recorded continuously for a period of 5 hours from morning 8:00 am to afternoon 1:00 pm to covering upto $120 \mathrm{~m}$ from the foot over bridge to towards Kurnool-Cadapa canal popularly known as K-C canal to assess the volume, speeds, trajectories, and lane change behavior of drivers of different types of vehicles.

For analysis of traffic characteristics the peak 15 minutes data were considered. And the total length of road stretch is divided into four parts, each part as $30 \mathrm{~m}$. This is done to know the accurate values of vehicle trajectories and lane changing behavior of driver. The vehicle trajectories, flow, speed were extracted using Traffic Data Extractor, for every 0.5 second resolution. To minimize the error of parallax the video is cropped and the lateral movements corresponding to the longitudinal distance of the vehicle were reported. Methodology of study is shown in fig. 3

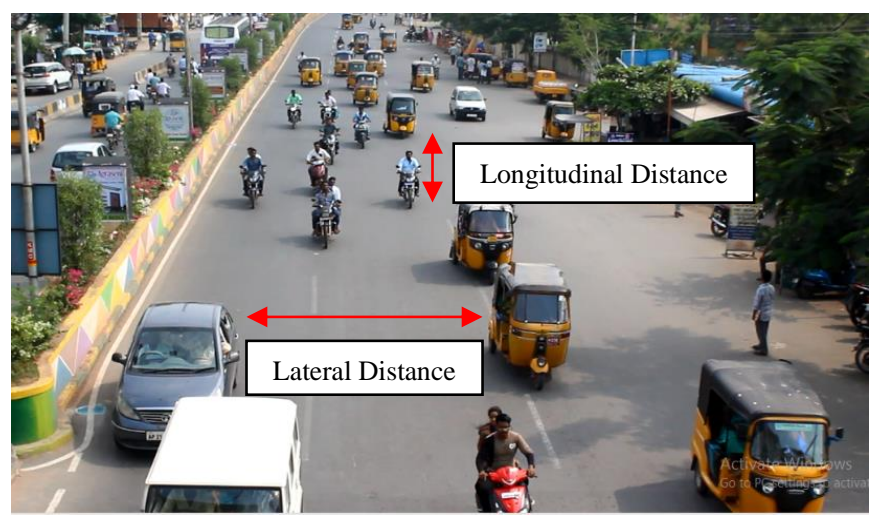

Fig.1: Screen shot of study area

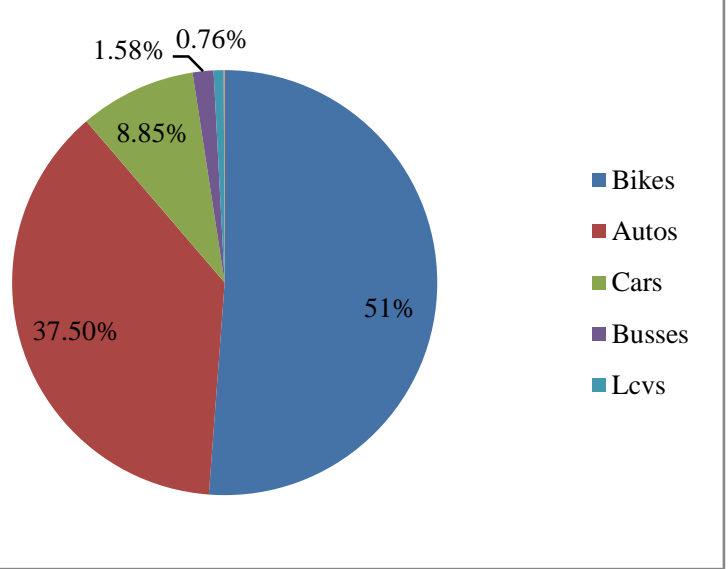

Fig.2: Proportion of vehicles

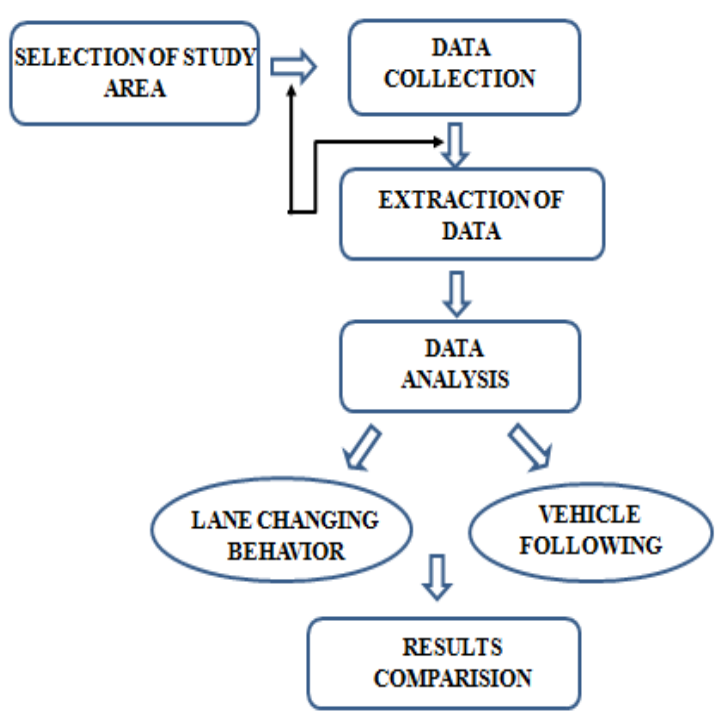

Fig. 3: Study Methodology 


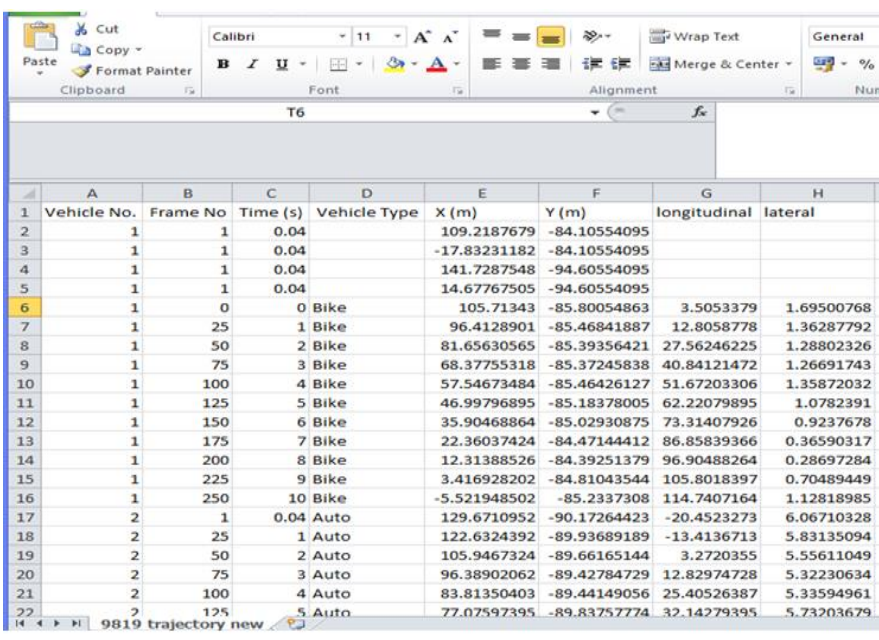

Fig.4: Trajectory Data Extraction in TDE

Table1: Volume, Observed vehicles and Instantaneous speeds of vehicles

\begin{tabular}{|c|c|c|c|c|c|c|}
\hline \multirow[t]{2}{*}{$\begin{array}{l}\text { Type of } \\
\text { vehicle }\end{array}$} & \multirow[t]{2}{*}{$\begin{array}{c}\text { Traffic } \\
\text { Volume(veh/hr) }\end{array}$} & \multirow[t]{2}{*}{$\begin{array}{c}\text { Observed } \\
\text { Vehicles }\end{array}$} & \multicolumn{4}{|c|}{$\begin{array}{c}\text { Instantaneous speeds of } \\
\text { vehicles(kmph) }\end{array}$} \\
\hline & & & Min & Max & Mean & St.De \\
\hline $\begin{array}{l}\text { Two } \\
\text { wheeler }\end{array}$ & 2454 & 1271 & 4.71 & 77.14 & 39.60 & 8.88 \\
\hline Auto & 1803 & 802 & 7.47 & 59.23 & 35.97 & 6.78 \\
\hline Car & 425 & 294 & 6.88 & 71.05 & 40.03 & 9.29 \\
\hline Bus & 76 & 71 & 8.28 & 72.97 & 42.05 & 9.72 \\
\hline LCV & 37 & 32 & 5.42 & 48.21 & 33.07 & 7.23 \\
\hline Average & 800 & - & 6.55 & 65.72 & 38.14 & 8.38 \\
\hline
\end{tabular}

The traffic video was taken for a period of 5 hours, from morning 8:00 am to mid afternoon of 1:00 pm to cover the peak and off peak traffic data. For analysis of traffic characteristics the peak 15 minutes data between 9:45 am to 10:00 am were considered. And Traffic flow, speed and densities were reported for 1 minute accuracy. Table 1 presents summary statistics of the traffic flow characteristics in the longitudinal direction. The reported speeds are for instantaneous values. The total maximum traffic flow observed in the study section is 6,342 vehicles per hour (vph). The maximum density ( veh $/ \mathrm{km} / \mathrm{hr}$ ) was observed as 83.142 for bikes and 71.971 for autos followed by 11.874 for cars. The instantaneous speeds of all the vehicles vary from 0 to 23.81 $\mathrm{m} / \mathrm{sec}$. Average speeds of all vehicles observed as $38.14 \mathrm{kmph}$. The mean speed of the buses are highest $42.05 \mathrm{kmph}$ followed by cars $40.03 \mathrm{kmph}$. LCVs travel at very less speed $33.07 \mathrm{kmph}$. Cars and bikes have higher lateral speeds $1.982 \mathrm{~m} / \mathrm{s}$ and $1.307 \mathrm{~m} / \mathrm{s}$ compared with other vehicles due to their higher maneuverability. Buses and LCVs have minimum lateral speeds due to their size and less maneuverable ability.

\section{Longitudinal and lateral velocities}

The rate at which a vehicle covers distance is referred to as speed. The rate at which the vehicle's location shifts is known as velocity. The average speed is symbolic and can be perceived as a negative number when moving in the opposite direction. The average speed has no sense in terms of direction and can be zero or positive. The velocity goes negative in the opposite direction. When the velocity value is negative, the vehicle is travelling in the opposite direction. A negative lateral velocity means that the vehicles are heading to the left, while a positive lateral velocity indicates that they are moving to the right. Negative lateral velocities show the vehicle on the left side and positive lateral velocities reveal the vehicle on the right side. Since there was heavy traffic, the instantaneous speeds of all the vehicles were predetermined and found to be exactly equal. The vehicles are travelling at a speed of 38 $\mathrm{kmph}$. The average lateral velocity is $0.125 \mathrm{~m} / \mathrm{sec}$. It was observed that roughly $70 \%$ of all vehicles drive to the left side, with the remaining $30 \%$ heading to the right. The median on the right hand side is may be reason for this. The rise in lateral velocities tested higher on the left hand side, and the velocities for all types of vehicles are about the same. This may be attributed to increased vehicular friction as a result of increased traffic volume and congestion. The average longitudinal velocity, on the other hand, is $9.0 \mathrm{~m} / \mathrm{sec}$. On the road segment, the majority of vehicles were two-wheelers, followed by autos, vans, LCVs, and buses. The lateral and longitudinal velocities of various vehicle types are depicted in Figure 5.
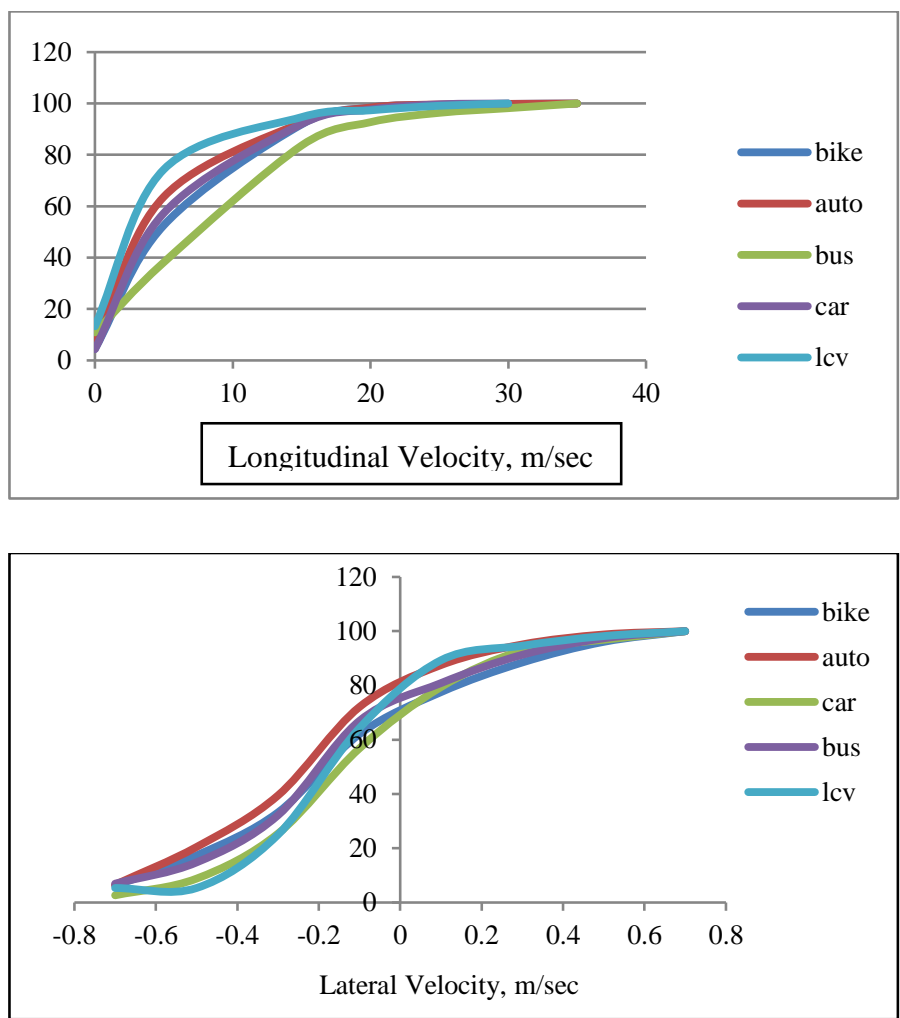

Fig.5: Longitudinal and Lateral velocities over the study section

\section{Time-Space diagram}

A time-space diagram is a practical approach to interpreting vehicle motion. It displays the vehicle's two- 
dimensional trajectory map. Time-space diagrams may be drawn for a single vehicle or a group of vehicles. Analysis processes on the position of the vehicle can be performed in terms of time by taking one vehicle at a time. The analysis produces a graph that depicts the vehicle's movement in one dimension in a normal, clear, and detailed manner. The location of the vehicle on the road in relation to time is represented in this study. These plots can easily visualise the following events, as well as the behaviour between and within the vehicles. This can then be summed up, and the vehicle's behaviour on the road can be calculated. The time-space diagram can be used to investigate the relationship between vehicle position and time in a traffic flow. The diagram represents the vehicle's travel along the longitudinal distance as a function of time. When all of the vehicles are considered for obtaining the measurements, the Time Space diagram in the upper part of Fig. 6 is shown. The Time-Space diagram in the lower part, on the other hand, is seen when only a limited number of vehicles are considered for obtaining the observation. Overlie is a phenomenon that happens when vehicles in the adjacent lane overcome each other. Twowheeled vehicles, autos, and cars were found to be more likely than others to take over the lead vehicle. Reduced speeds make the lines' slopes flatter, while increased speeds make the lines' slopes steeper. Both vehicles were in the same position at the same time when the curves crossed. Crossed curves are a sign of impending collisions unless passage is allowed.
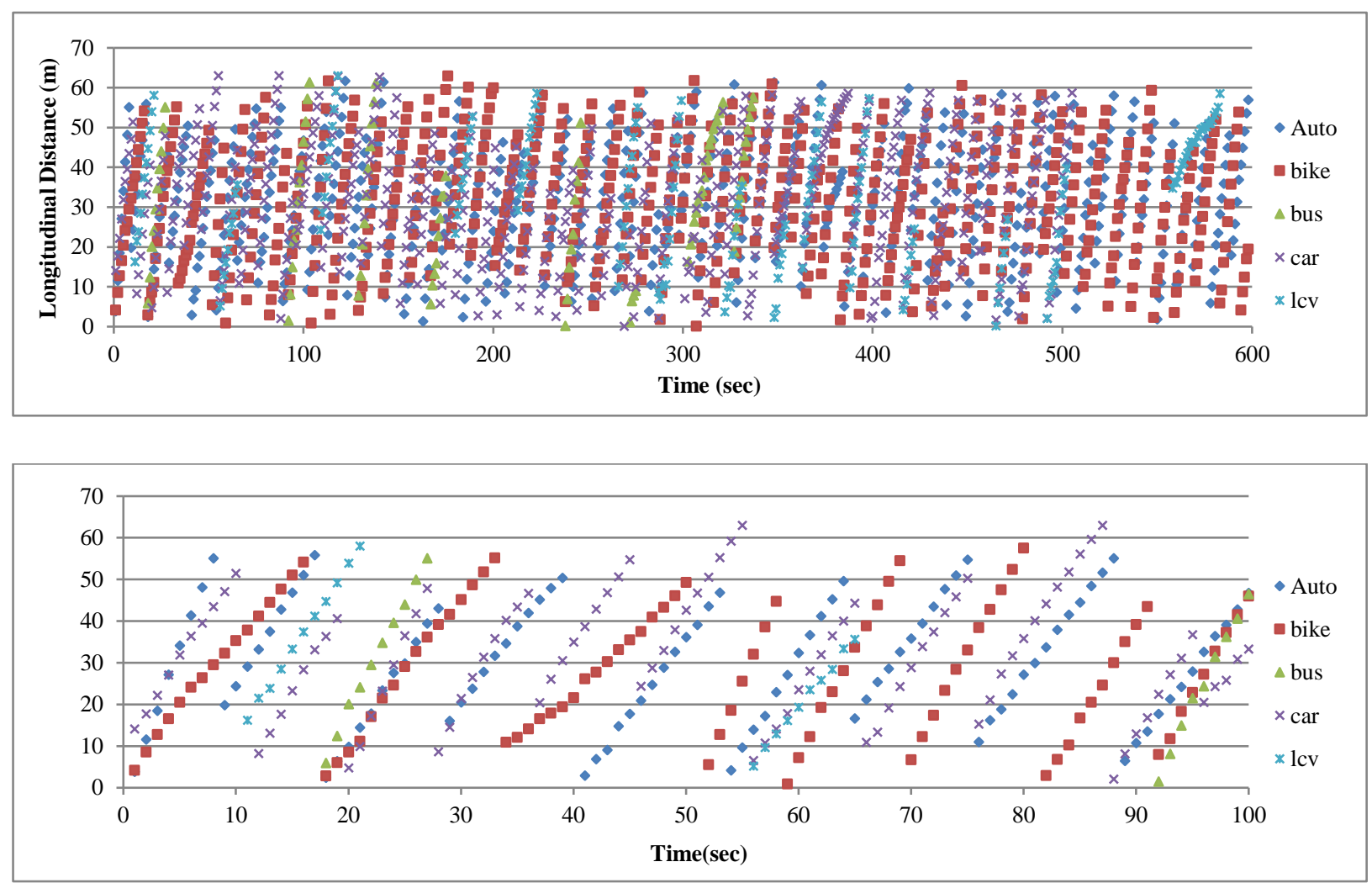

Fig 6: Time- space Diagram on selected stretch

\section{Lateral Position of Vehicles}

TDE was used to extract trajectory data in both longitudinal and lateral directions. The surveyed video files were loaded into the traffic data extractor user interface. The length and width of the road sections' traps is calculated by calculating the length and width of the designated rectangle portion on the software screen, which serves as road section calibration. Furthermore, the vehicle class of any given vehicle was entered into the software interface through observation. The vehicle can then be tracked by using the mouse pointer to click on the vehicle. Any vehicle in the traffic stream was monitored for the specified time period in a similar manner. The monitored data, in the form of image coordinates, was exported to excel files and then translated to real-world coordinates, along with vehicle trajectories. Vehicle trajectories were derived with a 1.0 second time resolution.

The lateral behavior was studied using extracted data by TDE. For this the median side was taken as origin with lane -1 followed by lane-2 and lane-3. The data after 3 lanes was not considered though the road is extended upto $1.5 \mathrm{~m}$, due to only $1.022 \%$ of the vehicles are observed while extracting the traffic volume data. Pedestrian volume and crossings are not considered due to the presence of foot over bridge. It is observed that a minimum gap from the median for bikes are $0.112 \mathrm{~m}$, where as the LCVs are moving at minimum distance of 0.574 from the median. Distributions of lateral displacements of various types of vehicles are shown in fig. From these charts, it is observed that most of the autos are 
moving in lane-2, buses, cars and LCVs are moving close to the median and bikes are moving almost all the width of the lane.

\section{Lateral Amplitude}

Nonetheless, little has been achieved in terms of vehicle lateral behaviour to date (the most essential criteria for the Indian state). Vehicle laterality is very high in comparison to other lane-disciplined countries since there is no lane control and mixed traffic conditions. Due to the lack of lane behaviour, a driver is forced to associate with vehicles not only longitudinally but also laterally with the surrounding vehicles

Over the study stretch, lateral amplitude was measured for each vehicle category to investigate lateral
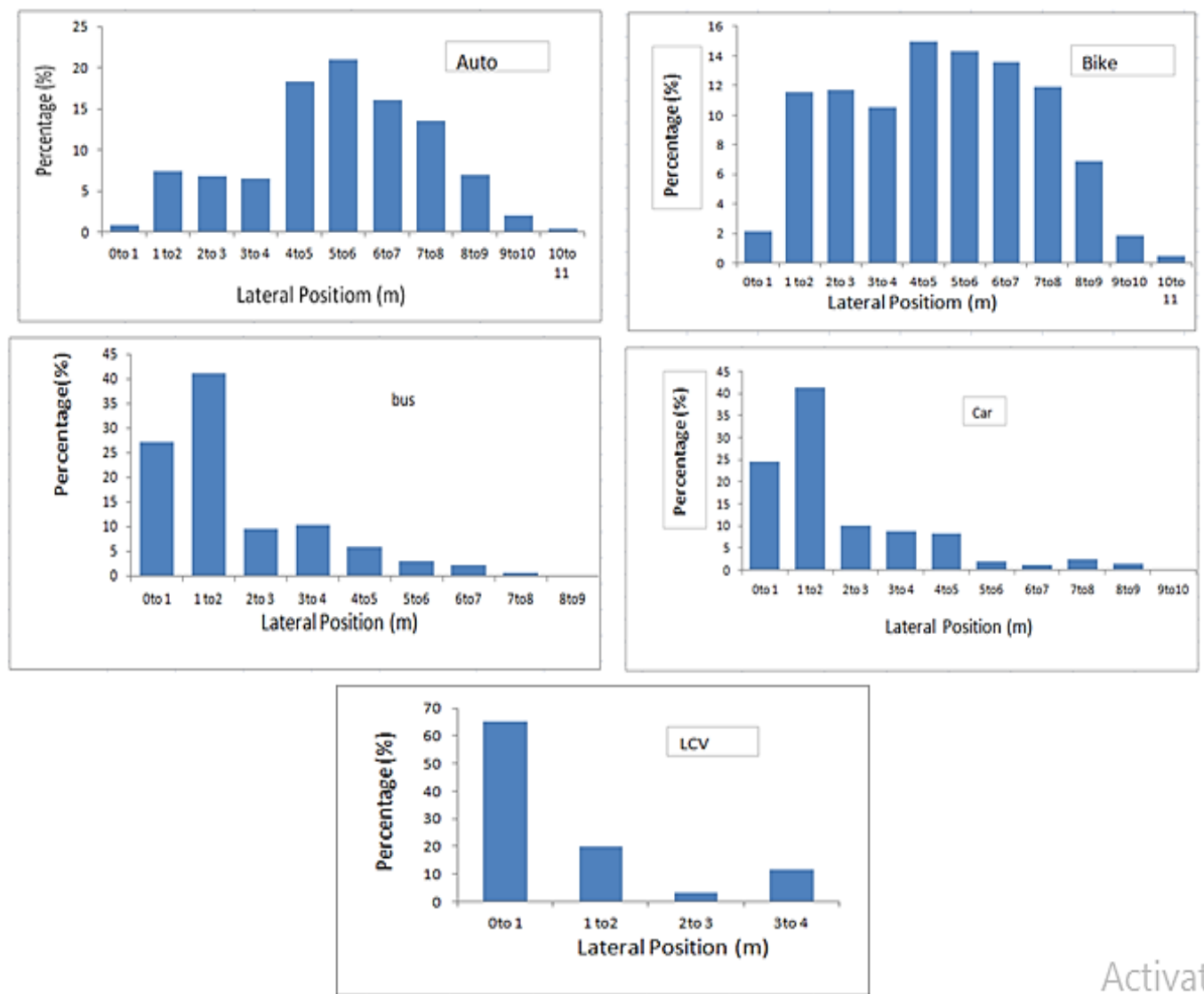

\section{Activat}

behaviour. The difference in maximum and minimum lateral positions over the study section (Narayana raju ), as shown in Figure, is the lateral amplitude of vehicles. In this regard, descriptive statistics for each vehicle category were calculated and shown in fig.8. According to careful observations, as the amount of traffic increases, the lateral weaving of all vehicles through the section increases. Since bikes and cars are larger than other vehicles in terms of static characteristics, they are able to move through other vehicles with little space between them. According to the lateral amplitude report, magnified vehicles are primarily responsible for non-lane based movement of other vehicles on the road section.

Fig.7: Lateral displacements of vehicles from median side

Table 2: Lateral Amplitude of vehicles over the study section

\begin{tabular}{|l|l|l|l|l|l|}
\hline Type of veh. & Min & Max & Range & Mean & St.De \\
\hline Auto & 0.19 & 6.19 & 3.67 & 1.42 & 0.90 \\
\hline Bike & 0.11 & 4.98 & 4.87 & 1.26 & 0.82 \\
\hline Bus & 0.33 & 2.30 & 1.97 & 1.02 & 0.61 \\
\hline Car & 0.12 & 4.55 & 4.46 & 0.91 & 0.85 \\
\hline LCV & 0.29 & 1.29 & 1.00 & 0.61 & 0.34 \\
\hline
\end{tabular}

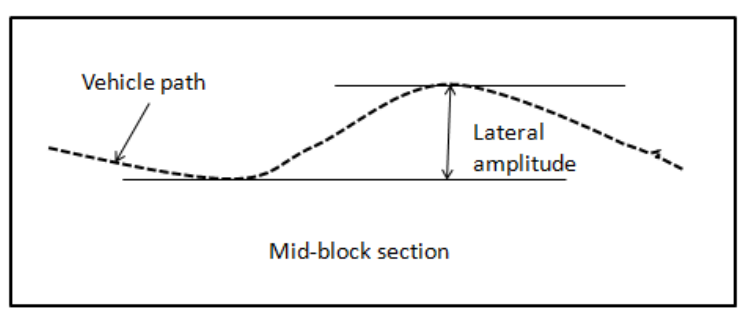




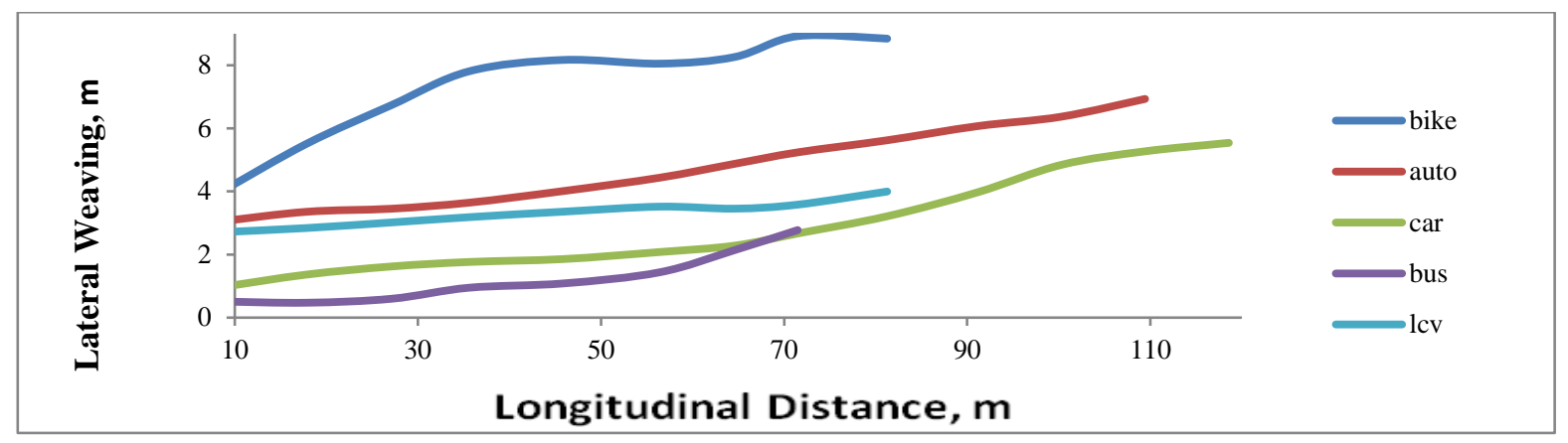

Fig.8: Lateral Amplitude of various types of vehicles

\section{Car Following Behavior}

Following a car or vehicle is one of the most complex perceptions that has existed for many years. The following vehicle is aligned with the leading vehicle ahead and the lag vehicle in mixed traffic conditions with wake or no lane discipline. There have been several studies on driver carfollowing behaviour. The relative distances and velocities of the leading and trailing vehicles were investigated in this report. The longitudinal distance between the lead and following vehicles travelling in the same direction and along the same line is known as relative distance. The relative speed refers to the difference between the vehicle speed and the leading vehicle speed. The higher the value of the following vehicle velocity, the higher the value of positive velocity, and vice versa. On the basis of relative distance and relative velocity, a new physical quantity known as time to collision was measured (TTC). TTC is a more popular safety component that is used in a number of car-following models to determine the risk of a rear-end collision. Rear-end accidents happen as a result of car-following processes. The TTC value is generally referred to as the amount of time it takes for two vehicles to crash if they continue at their current speeds and on a similar direction. As a result, a higher TTC value denotes a more secure situation. The distance between bike with any other vehicle is low when compared with other vehicles. On the other hand, the velocity of autos is higher as noticed. The obtained values of relative distance and velocities of various types of vehicles are tabulated in Table 3 and Table 4

Table.3: Relative distance of vehicles based on following driver

\begin{tabular}{|l|l|l|l|l|l|l|l|l|l|l|}
\hline \multirow{2}{*}{$\begin{array}{l}\text { Type of } \\
\text { Veh. }\end{array}$} & \multicolumn{3}{l|}{ Bike } & \multicolumn{2}{l|}{ Auto } & \multicolumn{2}{l|}{ Car } & \multicolumn{2}{l|}{ Bus } & \multicolumn{2}{l|}{ Lcv } \\
\cline { 2 - 12 }$y$ & Mean & St.de & Mean & St.De & Mean & St.De & Mean & St.De & Mean & St.De \\
\hline Bike & 9.26 & 6.13 & 10.30 & 6.04 & 9.97 & 6.08 & 14.7 & 7.57 & 16.21 & 8.03 \\
\hline Auto & 13.86 & 7.07 & 11.98 & 6.93 & 11.07 & 6.45 & 8.82 & 8.77 & 12.22 & 7.00 \\
\hline Car & 15.24 & 6.27 & 11.37 & 6.64 & 12.06 & 6.17 & 17.51 & 6.91 & 14.15 & 6.14 \\
\hline
\end{tabular}

Table.4: Relative velocities of vehicles based on following driver

\begin{tabular}{|l|l|l|l|l|l|}
\hline Type of veh & Min & Max & Mean & St.de & Range \\
\hline Bike & -19.23 & 29.16 & 0.26 & 5.25 & 48.39 \\
\hline Auto & -29.11 & 31.66 & -0.65 & 6.67 & 60.77 \\
\hline Car & -21.07 & 19.74 & -0.19 & 5.84 & 40.81 \\
\hline Bus & -16.19 & 18.30 & 1.72 & 7.28 & 34.49 \\
\hline LCV & -20.79 & 15.08 & -1.25 & 6.38 & 35.87 \\
\hline
\end{tabular}

\section{Time to Collision}

Based on relative distance and velocities the time to collision values are obtained. TTC depends on acceleration characteristics of following vehicle. The TTC thresholds were attained from k-means clustering analysis using python tool. The TTC risk level is divided into three categories which are high, medium and low. The risk level is high with a lower value of $0.213 \mathrm{sec}$ to a higher value of $15.69 \mathrm{sec}$ with an average of $3.09 \mathrm{sec} .92 .47 \%$ of the vehicles are observed to be in high risk level. The medium risk level ranges from 16.62 $\mathrm{sec}$ to $46.84 \mathrm{sec}$ with a mid range of $29.15 \mathrm{sec}$ simultaneously $5.34 \%$ of the vehicles are recorded to be at medium risk level. Lastly the least risk level begins at $58.42 \mathrm{sec}$ to $94.06 \mathrm{sec}$ with a mean of $78.07 \mathrm{sec}$ and only $2.19 \%$ of the vehicles' presence is noticed in the low risk level. The TTC values acquired are shown in Table5. As maximum number of vehicles is maintaining less relative distances and more relative velocities based on following type vehicle, there is a chance of rear end collision between lead and following vehicle.

Table.4: Time to Collision

\begin{tabular}{|l|c|c|c|c|c|}
\hline $\begin{array}{l}\text { Risk Level } \\
\text { (sec) }\end{array}$ & Min & Max & Mean & St.De & \% Risk \\
\hline High & 0.213 & 15.69 & 3.09 & 3.42 & 92.47 \\
\hline Medium & 16.62 & 46.84 & 29.15 & 9.57 & 5.34 \\
\hline Low & 58.42 & 94.06 & 78.07 & 12.43 & 2.19 \\
\hline
\end{tabular}




\section{Conclusions}

An attempt was made in this document to examine the traffic characteristics of mixed traffic. In an urban midblock road segment of Kurnool City in Andhra Pradesh, a comprehensive collection of vehicle trajectory data was collected to evaluate longitudinal or lateral features. Weak lane control is a critical aspect of the mixed traffic. The study showed that vehicles generally and in particular motorcycles and cars travel significantly laterally within the mixed stream. The following general conclusions have been drawn on the basis of the observing data research and interpretation.

- Bikes, cars and buses compare with LCVs, with a longitudinal flow characteristic that suggest that speeds and accelerations are high. This may be because LCV's are poorly dynamic.

- Most of all LCV and buses move very closely to the middle lane and choose the first. Since most automobiles and motorcycles choose second and third routes

- The lateral amplitude of vehicles indicates that the lateral movement of motorcycles and Autos are high, because of their smaller dimension relative to other vehicles.

- The acquired TTC-values indicate that there is a risk of a rear-end collision between the leading and the subsequent vehicle based on the following model vehicle that maximum vehicles retain lower relative distances and more relative speeds.

\section{References}

[1] Narayanaraju, Pallavkumar, Aayushjain "Application of Trajectory data for investigating the vehicle behavior in mixed traffic environment", Transportation Research Record, Journal of the Transportation Research Board, July 2018

[2] Narayanaraju, Pallavkumar, Aayushjain , "Examining smoothening techniques for developing vehicular trajectory data under heterogeneous conditions" Journal of the Eastern Asia Society for Transportation Studies, Vol.12, 2017

[3] Venkatesan Kanagaraj, Gowri Asaithambi, Tomer Toledo, and Tzu-Chang Lee, "Trajectory Data and Flow Characteristic of Mixed Traffic". Transportation Research Record: Journal of the Transportation Research Board,No. 2491, Transportation Research Board, Washington, D.C., 2015, pp. 1-11.

[4] Shen Li, Qiaojun Xiang, YongfengMa , XinGu and Han Li , "Crash Risk Prediction Modelling Based on the Traffic Conflict Technique and a Microscopic Simulation for Freeway Interchange Merging Areas". International Journal of environmental research and public health.

[5] V.S.H .Bangarraju, K.V.R. Ravi Shankar, Tom V Mathew, "Analysis of lateral distance keepingbehavior in mixed traffic conditions with little lane discipline" International Journal for Traffic and Transport Engineering, 2016, 6(4): 431 - 443.

[6] MohdErwanSanik, Nor Baizura Hamid, Ahmad Hakimi Mat Nor, Joewono Prasetijo, Mohd Safberi Che Ani and FajaruddinMustakim , "Drivers lane changing behaviour at urban intersection by using gap acceptance approach" ARPN Journal of Engineering and Applied Sciences.

[7] Geethi Mukta Mahapatra, Akhilesh kumar, Mourya , "Study of vehicles lateral movement on non lane discipline traffic stream on a straight road" Published by Elsevier Ltd.Selection and peer-review under responsibility of International Scientific Committee. .

[8] Prof. K. V. Krishna Rao, Prof. Tom V. Mathew, "Traffic Data Extractor", Development of Indian Highway, Capacity Manual.

[9] Arne Kesting, Martin Treiber, and Dirk Helbing, "General Lane-Changing Model MOBIL for CarFollowing Models Article", Transportation Research Record Journal of the Transportation Research Board.

[10] K. Durga Rani, K.Venkata Subbaiah, C.N.V. Satyanarayana Reddy, and S.S.S.V. Gopala Raju, "A Simulation Study on Overtakings and Lane Changing Behaviour On An Urban Road Corridor", International Journal of Engineering Research and Technology, ISSN 0974-3154 Volume 3, Number 3 (2010), pp. 691 - 698, International Research Publication House.

[11] Chai , Y.D.Wong, "Micro-simulation of vehicle conflicts involving right-turn vehicles at signalized intersections based on cellular automata", Centre for Infrastructure Systems, Nanyang Technological University, 40 Nanyang Avenue, 639798, Singapore

[12] Mahendrakumar Metkari a, Anuj Budhkarb, Akhilesh Kumar Maurya, "Development of Simulation Model for Heterogeneous Traffic with No Lane Discipline", 2nd Conference of Transportation Research Group of India (2nd CTRG).

[13] Mallikarjuna, C., A. Phanindra, and K. R. Rao. "Traffic Data Collection Under Mixed Traffic Conditions Using Video Image Processing",Journal of Transportation Engineering, Vol. 135, No. 4, 2009, pp. 174- 182.

[14] Toledo, T., H. N. Koutsopoulos, and M. Ben-Akiva, “ Modeling Integrated Lane-Changing Behavior", Transportation Research Record: Journal of the Transportation Research Board, 2003.1857: 30-38.

[15] Metkari, M.; Budhkar, A.; Maurya, A. K. 2013, "Development of Simulation Model for Heterogeneous Traffic with No Lane Discipline", Procedia-Social and Behavioural Sciences, 104: 360-369.

[16] Santel, G. 2010., "Lateral driving behaviour", In Proceedings of 10th Swiss Transport Research Conference, ETH Zurich, Monte Verita, 1-26.

[17] Sangole, J. P., and G. R. Patil, "Modeling Vehicle Group Gap Acceptance at Uncontrolled T-Intersections in Indian Traffic", Presented at 93rd Annual Meeting of the Transportation Research Board, Washington, D.C., 2014.

[18] Toledo, T., H. N. Koutsopoulos, and K. I. Ahmed, "Estimation of Vehicle Trajectories with Locally Weighted Regression", In Transportation Research Record: Journal of the Transportation Research Board, No. 1999, Transportation Research Board of the National Academies, Washington, D.C., 2007, pp. 161169. 
[19] Munigety, C. R., V. Vivek, and T. V. Mathew. "Semiautomated Tool for Extraction of Microlevel Traffic Data from Video graphic Survey". Transportation Research Record: Journal of the Transportation Research Board, No. 2443, Transportation Research Board of the National Academies, Washington, D.C., 2014, pp. 88-9

[20] Mathew, T. V., C. R. Munigety, and A. Bajpai. 2013. "A strip-based approach for the simulation of mixed traffic conditions." J.Comput. Civ. Eng. 29 (5): 04014069. https://doi.org/10.1061/(ASCE)CP.1943 5487.0000378 . 